\title{
Personalized Modeling Pipeline for Left Atrial Electromechanics
}

\author{
Thomas E Fastl ${ }^{1}$, Catalina Tobon-Gomez ${ }^{1}$, William A Crozier ${ }^{2}$, John Whitaker ${ }^{1,3}$ \\ Ronak Rajani ${ }^{1,3}$, Karen P McCarthy ${ }^{4}$, Damian Sanchez-Quintana ${ }^{5}$ \\ Siew Y Ho ${ }^{4}$, Mark D O'Neill ${ }^{1,3}$, Gernot Plank ${ }^{2}$, Martin J Bishop ${ }^{1}$, Steven A Niederer ${ }^{1}$ \\ ${ }^{1}$ Department of Biomedical Engineering, King's College London, London, United Kingdom \\ ${ }^{2}$ Institute of Biophysics, Medical University of Graz, Graz, Austria \\ ${ }^{3}$ Department of Cardiology, Guy's and St Thomas' Hospitals, London, United Kingdom \\ ${ }^{4}$ Cardiac Morphology Unit, Royal Brompton Hospital, London, United Kingdom \\ ${ }^{5}$ Department of Anatomy and Cell Biology, Universidad de Extremadura, Badajoz, Spain
}

\begin{abstract}
Atrial fibrillation $(A F)$ is a supraventricular tachyarrhythmia characterized by uncoordinated atrial activation with consequent deterioration of mechanical function. Personalized computational modeling provides a novel framework for integrating and interpreting the combined role of atrial electrophysiology and mechanics in AF development and sustenance. Coronary computed tomography angiography data were segmented using a threshold-based approach and the smoothed voxel representation was discretized into a high-resolution tetrahedral finite element (FE) mesh. To estimate the complex left atrial fiber architecture, individual fiber fields were generated according to morphological data on the endo- and epicardial surfaces based on local solutions of Laplace's equation and transmurally interpolated to all tetrahedral elements. Personalized geometrical models included the heterogeneous thickness distribution of the left atrial myocardium and subsequent discretization led to high-fidelity tetrahedral FE meshes. The novel algorithm for (automated) incorporation of the left atrial fiber architecture provided a realistic estimate of the atrial microstructure and was able to qualitatively capture all important fiber bundles. The established modeling pipeline provides a robust framework for the rapid development of personalized model cohorts and facilitates simulations of atrial electromechanics.
\end{abstract}

\section{Introduction}

Atrial fibrillation (AF) is a supraventricular tachyarrhythmia characterized by uncoordinated atrial activation with consequent deterioration of mechanical function. Affecting an estimated 33 million people worldwide [1], $\mathrm{AF}$ is the most common type of arrhythmia increasing the long-term risk of other cardiovascular diseases. Although cases of lone AF exist, this medical condition is often secondary to hypertension and heart failure, both pathologies impacting the mechanical loading conditions of the atria.

Personalized computational modeling provides a novel framework for integrating and interpreting the combined role of atrial electrophysiology and mechanics in the development and progression of AF. Computational studies in the atria have demonstrated the influence of the structural anisotropy on electrophysiology or finite elasticity for different species substantiating the importance of the atrial fiber architecture in finite element (FE) simulations [2,3]. Significant limitations apply to in vivo imaging of the atrial fiber architecture, therefore, alternative approaches have been developed to inform personalized models of the atria.

The majority of atrial modeling studies have incorporated the fiber architecture using rule-based approaches to qualitatively represent the observed fiber morphology in the literature. Due to the complex nature of the atrial fiber architecture, however, the applied rules have been manually attributed to specific atrial regions and structures [4-6]. Although the individual methods have led to adequate representations of the atrial fiber distribution, these approaches are not suitable for large patient cohorts. To generate personalized computational models of the atria including the fiber orientation for multiple patient geometries, a variety of rule-based semi-automated approaches have been proposed $[2,3,7]$. While these methods have estimated the atrial fiber architecture with reasonable detail, the degree of manual intervention leads to increased intraand interobserver variability.

A novel modeling pipeline to generate detailed personalized computational models for left atrial electromechanics is introduced in this study requiring a minimum degree of manual intervention. This facilitates the consistent development of atrial FE models including the complex fiber architecture using clinical data from large patient cohorts. 


\section{Methods}

The novel (automated) modeling pipeline for generating detailed personalized computational models of the left atrium (LA) is illustrated in Fig. 1. Retrospective analysis of a single patient (female; 55 years) who underwent a clinically indicated coronary computed tomography angiography (CTA) to exclude coronary artery disease was performed to exercise the proposed atrial modeling pipeline.

\subsection{Anatomical Imaging}

Coronary CTA data were acquired at Guy's and St Thomas' NHS Foundation Trust using a Philips 256 iCT scanner (Philips Healthcare, Amsterdam, Netherlands). The acquired cardiac CTA data were reconstructed using an iterative reconstruction (iDose level 4) with $0.8 \mathrm{~mm}$ slice thickness, $0.4 \mathrm{~mm}$ slice increment, $250 \mathrm{~mm}$ field of view, $512 \times 512$ matrix, and a smooth reconstruction kernel.

\subsection{Image Segmentation}

Based on the assumption of comparable radiodensities in the atrial and ventricular myocardium [8], a regionally tagged segmentation of the LA was generated using the software package Seg3D2. Within the acquired image data layer, 3 sample regions were selected in both the left ventricular myocardium and the left atrial contrast bolus, for which combined mean values and standard deviations were calculated. The viable left atrial myocardium and the left atrial contrast bolus were thresholded utilizing their corresponding statistical sample mean value increased or reduced by 3 times the standard deviation, respectively. The continuous transition between the left atrial myocardium and the left atrial contrast bolus required a common threshold value chosen as the average of both statistical mean values of the sample regions. Initial dilation of the left atrial contrast bolus by 1 voxel was performed to obtain a minimum wall thickness. The subsequent iterative dilations combined with logical operations on the viable atrial myocardium provided the segmentation of the left atrial wall. Standardized mitral valve extraction as well as pulmonary vein truncation was performed using implemented postprocessing tools.

A combined smoothing and upsampling method was applied on the anatomically tagged voxel representation attenuating possible staircase effects and providing a spatial resolution suitable for computational FE modeling [9]. Smoothing was achieved by minimization of a high-order penalty restricting the maximum displacement of the reference surface to $50 \%$ of the initial voxel size. The resulting surface was resampled providing an image segmentation with a consistently isotropic voxel resolution.

\subsection{Mesh Generation}

A high-fidelity and high-resolution mesh of the LA was created using the Octree-based mesh generation software Tarantula (CAE Software Solutions, Eggenburg, Austria), which builds unstructured, boundary fitted, locally refined tetrahedral meshes and maps classification tags from the input segmentation onto the generated mesh. Identification of the endo- and epicardium including the corresponding surface meshes of the LA relied on a breath-first search algorithm implemented in MATLAB (The MathWorks, Inc., Natick, United States).

\subsection{Image Registration}

A major advantage of the proposed method to generate detailed personalized computational models of the LA over existing ones is the automated transfer of predefined landmarks from an average atrial geometry to the personalized atrial geometry. This average atrial geometry was generated by combination of 30 magnetic resonance imaging datasets and manual segmentation of the endocardium.

Endo- and epicardial surface meshes were decimated to approximately $10 \%$ of the reference element number using the Computational Geometry Algorithms Library facilitating a tractable image registration. The resulting surface meshes were manually transformed to match the average atrial geometry and subsequently registered using the software Deformetrica. A nearest neighbor approach was applied to transfer 122 predefined landmarks on the average atrial geometry to the corresponding decimated and registered surface mesh. During the image registration process the mesh topology was maintained, hence, a final nearest neighbor transfer of the landmarks from the decimated to the personalized atrial surface mesh provided their location. An additional 66 landmarks were calculated for the pulmonary veins, the mitral valve, the interatrial septum, and the left atrial appendage. Minor manual adjustment of the points around the left atrial appendage was performed where necessary as a consequence of the large structural variability.

\subsection{Surface Fiber Generation}

Landmarks on the left atrial surfaces constituted a predefined network of 272 auxiliary lines, which subdivided both surfaces into 151 atrial regions. The auxiliary lines were calculated using an implementation of the Dijkstra algorithm applied on preselected corridors utilizing the geodesic paths between the corresponding landmarks. Then, a region growing algorithm identified the atrial regions, on which Laplace's equation

$$
\nabla^{2} u=0
$$



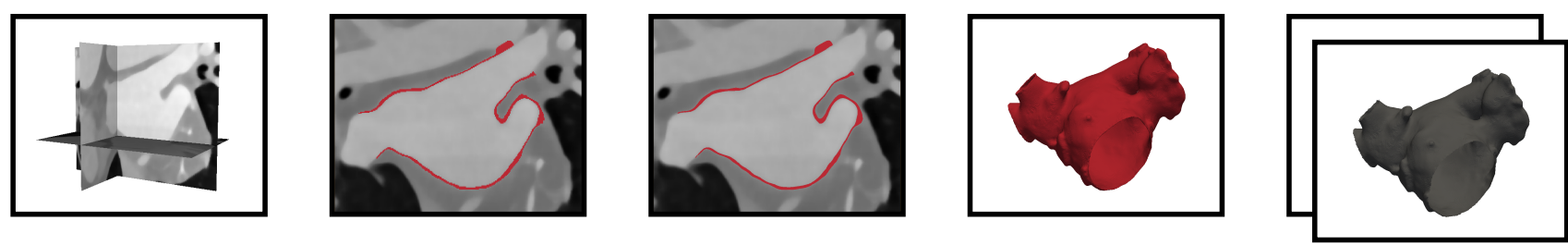

Mesh Generation

Fiber Generation

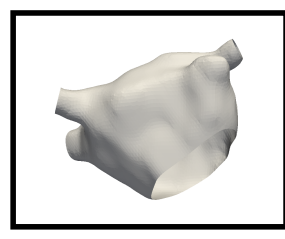

Atrial Atlas

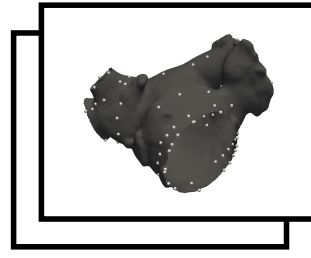

Landmark Transfer

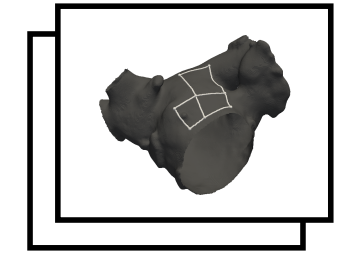

Auxillary Lines|Regions

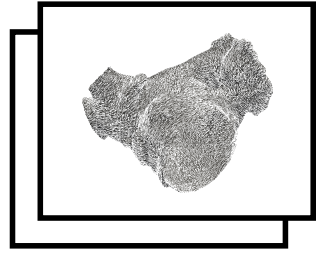

Surface Fibers

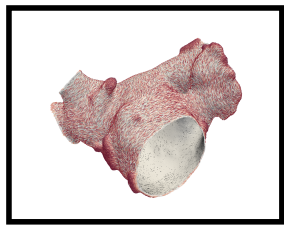

Volume Fibers

Figure 1. Personalized modeling pipeline for left atrial electromechanics separated into the mesh generation phase (top) and the subsequently performed fiber generation phase (bottom). Individual boxes illustrate a representative generation processes, while two boxes indicate processes on multiple surfaces, i.e., endo- and epicardium.

was solved within the Cardiac Arrhythmia Research Package using boundary conditions applied to bordering auxiliary lines, i.e.,

$$
\left.u\right|_{\partial \Omega_{\mathrm{I}}}=0,\left.\quad u\right|_{\partial \Omega_{\mathrm{II}}}=1,\left.\quad \frac{\partial u}{\partial n}\right|_{\partial \Omega_{\mathrm{R}}}=0 .
$$

The normalized gradient of the obtained scalar field $\nabla u /\|\nabla u\|$ evaluated at the surface triangle centers represented the modeled fiber orientation and estimated the morphologically observed atrial fiber architecture. The calculated fiber vectors were combined and complemented with a synthetic fiber orientation to obtain a full representation of the personalized fiber field on the endo- and epicardial surfaces of the LA. Interregional fiber smoothing using an orientation-based neighborhood approach was applied to triangular elements in the proximity of the auxiliary lines as well as elements with synthetic fiber orientations. Finally, out-of-plane fibers due to the smoothing procedure were projected onto the tangential plane of the atrial surface followed by vector normalization.

\subsection{Transmural Fiber Generation}

Fiber direction vectors for tetrahedral elements were obtained by transmural interpolation of the estimated surface fiber field according to a correspondence map. Therefore, (1) was solved on the tetrahedral FE mesh with boundary conditions in (2) applied to the endocardium $\left(\Omega_{\mathrm{I}}\right)$ and epicardium $\left(\Omega_{\mathrm{II}}\right)$. Starting from the tetrahedral element center, endo- and epicardial surface triangles were identified by tracking the unit field line normals evaluated as $\nabla u /\|\nabla u\|$. An element average of the nodal solutions $u$ was calculated to separate the atrial myocardium according to

$$
\Omega(u)= \begin{cases}\Omega_{\mathrm{ED}} & \text { if } u \leq 0.5 \\ \Omega_{\mathrm{EP}} & \text { if } u>0.5\end{cases}
$$

in distinct layers. The fiber direction in all tetrahedral elements was assigned based on the estimated direction vector of the surface triangle according to the generated correspondence map.

\section{Results}

Acquired coronary CTA images were retrospectively analyzed and the corresponding threshold ranges for the viable left atrial myocardium and the left atrial contrast bolus were calculated as $89.04-314.27 \mathrm{HU}$ and 314.27 $566.27 \mathrm{HU}$, respectively. The tetrahedral FE mesh consisted of 51543435 elements scaffolded by 9697385 vertices, while the maximum tetrahedral element edge length was determined to be $204.12 \mu \mathrm{m}$. Individual images in Fig. 2 display the atrial fiber orientation in the most prominent regions of the LA, the anterior wall including the Bachmann's bundle, the posterior wall, the pulmonary veins, and the mitral valve annulus. The estimated fiber architecture was validated by anatomical experts and qualitatively matched a comprehensive set of morphological data.

\section{Discussion}

The (automated) modeling pipeline generates highfidelity and high-resolution tetrahedral FE meshes compat- 


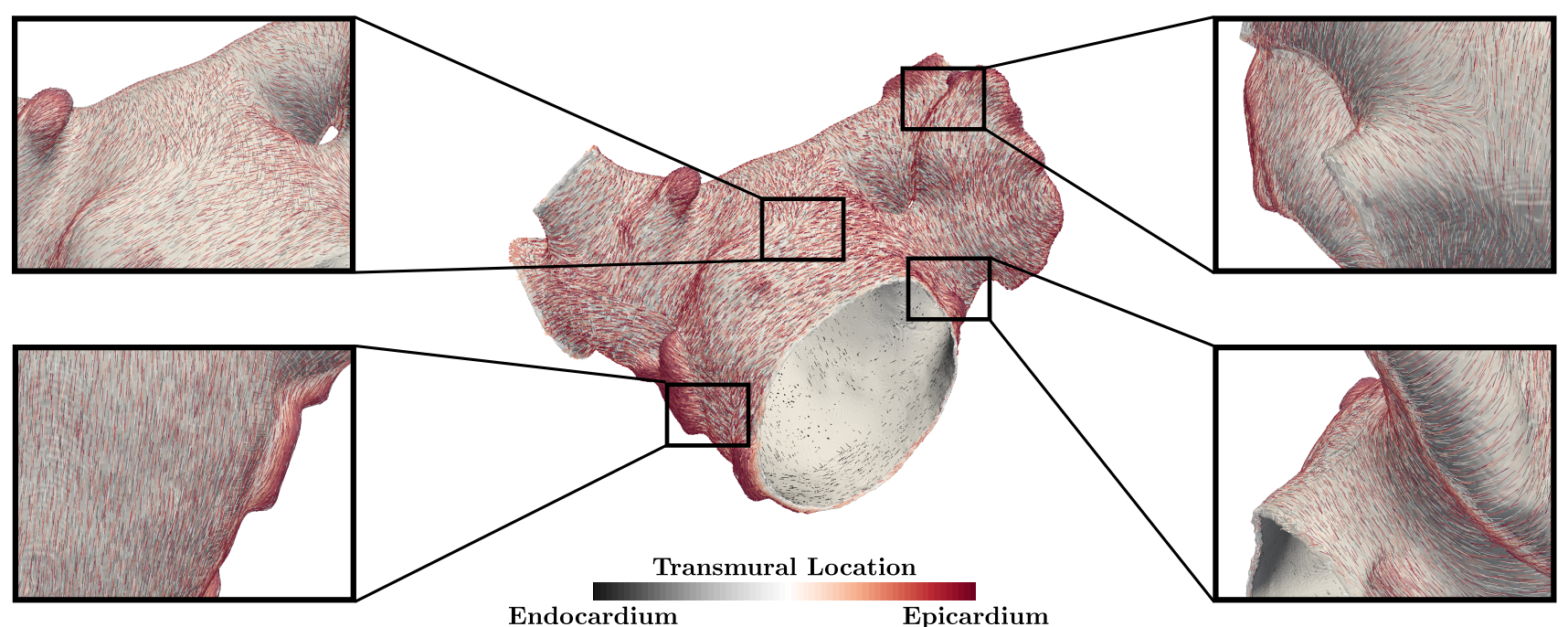

Figure 2. Generated left atrial fiber orientation at different locations represented using color-coded cylinders. Zoomed inlays show the transmural fiber architecture in the anterior wall including the Bachmann's bundle (top left), the posterior wall (bottom left), the pulmonary veins (top right), and the mitral valve annulus (bottom right).

ible with the requirements for electrophysiology and mechanics. The novel approach provides reproducible and morphologically accurate representations of the fiber architecture in the LA for large patient cohorts. Another major advantage is the potential for application of the algorithm to generate both shell and volume models, while in the latter case the transmural fiber variation can be interpolated.

\section{Acknowledgements}

The authors greatly acknowledge the valuable technical support of Anton J Prassl on the mesh generation software.

\section{References}

[1] Rahman F, Kwan GF, Benjamin EJ. Global epidemiology of atrial fibrillation. Nat Rev Cardiol 2014;11(11):639-54.

[2] Krueger MW, Schmidt V, Tobón C, Weber FM, Lorenz C, Keller DUJ, Barschdorf H, Burdumy M, Neher P, Plank G, Rhode K, Seemann G, Sanchez-Quintana D, Saiz J, Razavi $\mathrm{R}$, Dössel O. Modeling atrial fiber orientation in patientspecific geometries: A semi-automatic rule-based approach. In Metaxas DN, Axel L (eds.), Functional Imaging and Modeling of the Heart. Berlin, Heidelberg: Springer, 2011; 22332.

[3] Satriano A, Bellini C, Vigmond EJ, Di Martino ES. A feature-based morphing methodology for computationally modeled biological structures applied to left atrial fiber directions. J Biomed Eng 2013;135(3):31001.

[4] Seemann G, Höper C, Sachse FB, Dössel O, Holden AV, Zhang H. Heterogeneous three-dimensional anatomical and electrophysiological model of human atria. Philos Trans A Math Phys Eng Sci 2006;364(1843):1465-81.
[5] Gonzales MJ, Sturgeon G, Krishnamurthy A, Hake J, Jonas R, Stark P, Rappel WJ, Narayan SM, Zhang Y, Segars WP, McCulloch AD. A three-dimensional finite element model of human atrial anatomy: New methods for cubic Hermite meshes with extraordinary vertices. Med Image Anal 2013; 17(5):525-37.

[6] Ferrer A, Sebastián R, Sánchez-Quintana D, Rodríguez JF, Godoy EJ, Martínez L, Saiz J. Detailed anatomical and electrophysiological models of human atria and torso for the simulation of atrial activation. PLoS One 2015; 10(11):e0141573.

[7] Labarthe S, Coudiere Y, Henry J, Cochet H. A semiautomatic method to construct atrial fibre structures: A tool for atrial simulations. Comput Cardiol 2012;39:881-4.

[8] Bishop M, Rajani R, Plank G, Gaddum N, Carr-White G, Wright M, O'Neill M, Niederer S. Three-dimensional atrial wall thickness maps to inform catheter ablation procedures for atrial fibrillation. Europace 2016;18(3):376-83.

[9] Crozier A, Augustin CM, Neic A, Prassl AJ, Holler M, Fastl TE, Hennemuth A, Bredies K, Kuehne T, Bishop MJ, Niederer SA, Plank G. Image-based personalization of cardiac anatomy for coupled electromechanical modeling. Ann Biomed Eng 2016;44(1):58-70.

Address for correspondence:

Thomas E Fastl

St Thomas' Hospital

King's College London

London, SE1 7EH

United Kingdom

thomas.fastl@kcl.ac.uk 